\title{
Utility of Three-Dimensional Volume Rendering Images Using Electron-Beam Computed Tomography to Evaluate Possible Causes of Ischemia From an Anomalous Origin of the Right Coronary Artery From the Left Sinus of Valsalva
}

\author{
Nobusada Funabashi, MD; Yoshiki Kobayashi, MD*; \\ Geoffrey D. Rubin, MD**
}

\begin{abstract}
The present study evaluated the usefulness of 3-dimensional volume rendering (VR) images using electron-beam computed tomography (EBCT) in determining the possible causes of ischemia resulting from the anomalous origin of the right coronary artery (RCA) from the left sinus of Valsalva, which coursed between the ascending aorta and pulmonary trunk. Such anomalies could cause ischemia or sudden death without obstructive coronary artery disease. The suggested mechanism is either compression causing closure of the slit-like orifice of the anomalous artery as the aorta dilates with exertion or compression of the anomalous artery by the aorta and pulmonary trunk as it courses between these 2 arteries, which dilate with exercise. A 17-year-old male underwent EBCT coupled with a 100-ml intravenous injection of iodinated contrast medium. Data were reconstructed into 3-dimensional images through VR to evaluate the shape of the orifice and the spatial relationship of the RCA, ascending aorta and pulmonary trunk. Perspective VR showed the shape of the orifice of the left main trunk, which was not slit-like, and cut-plane VR showed the spatial relationship of both the lumen and the surface of the RCA, ascending aorta and pulmonary trunk, providing information on whether the ascending aorta or pulmonary trunk would compress the RCA and cause ischemia. (Jpn Circ J 2001; 65: 575-578)
\end{abstract}

Key Words: Anomalous origin of coronary artery; Electron-beam computed tomography; Ischemic mechanism; Volume rendering

A

berrant right coronary artery (RCA) originating from the left sinus of Valsalva is an infrequent coronary anomaly reported to occur in $0.19 \%^{1}$ and $0.17 \%{ }^{2}$ of patients undergoing diagnostic coronary angiography. Several reports have described an RCA originating from the left sinus of Valsalva and then coursing between the ascending aorta and the pulmonary trunk (PT), and this anomaly has been speculated as a cause of ischemia or sudden death in the absence of obstructive coronary artery disease $!^{-7}$ Three possibilities exist for the ischemic mechanism: the slit-like orifice of the anomalous coronary artery is compressed to the point of closure or near closure as the aorta dilates with exertion; the anomalous artery itself is compressed by the aorta and the PT as it courses between these 2 arteries, which dilate with exercise; or both of these factors? The present study used 3-dimensional volume rendering (VR) images from electron-beam computed tomography (EBCT) to investigate the possible causes of ischemia from such an anomalous RCA, as well as investigated the course of the RCA. For these purposes, information on the shape of the orifice of the left main trunk, the

(Received November 9, 2000; revised manuscript received January 30, 2001; accepted February 7, 2001)

The Third Department of Internal Medicine, Chiba University, Chiba, *The First Department of Internal Medicine, Osaka City University, Osaka, Japan and **Department of Radiology, Stanford University School of Medicine, Stanford, CA, USA

Mailing address: Nobusada Funabashi, MD, The Third Department of Internal Medicine, Chiba University, 1-8-1 Inohana, Chuo-ku, Chiba-city, Chiba 260-8670, Japan spatial relationship of the lumen and the surface of the RCA, ascending aorta and the PT, and the angle of the bend of the bifurcation of the left main trunk (angle of the left main trunk to the RCA) would be useful.
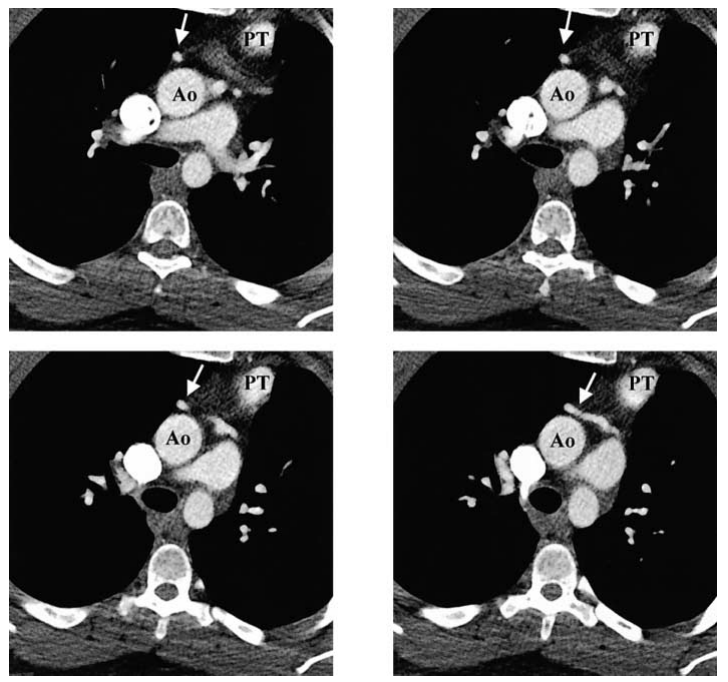

Fig 1. Axial source images of enhanced electron-beam computed tomography showing the right coronary artery (arrow) originating from the left sinus of Valsalva with the left main trunk and coursing between the ascending aorta (Ao) and the pulmonary trunk (PT). The angle of the bend at the bifurcation of the left main trunk (angle of the left main trunk to the right coronary artery) is sharp. 


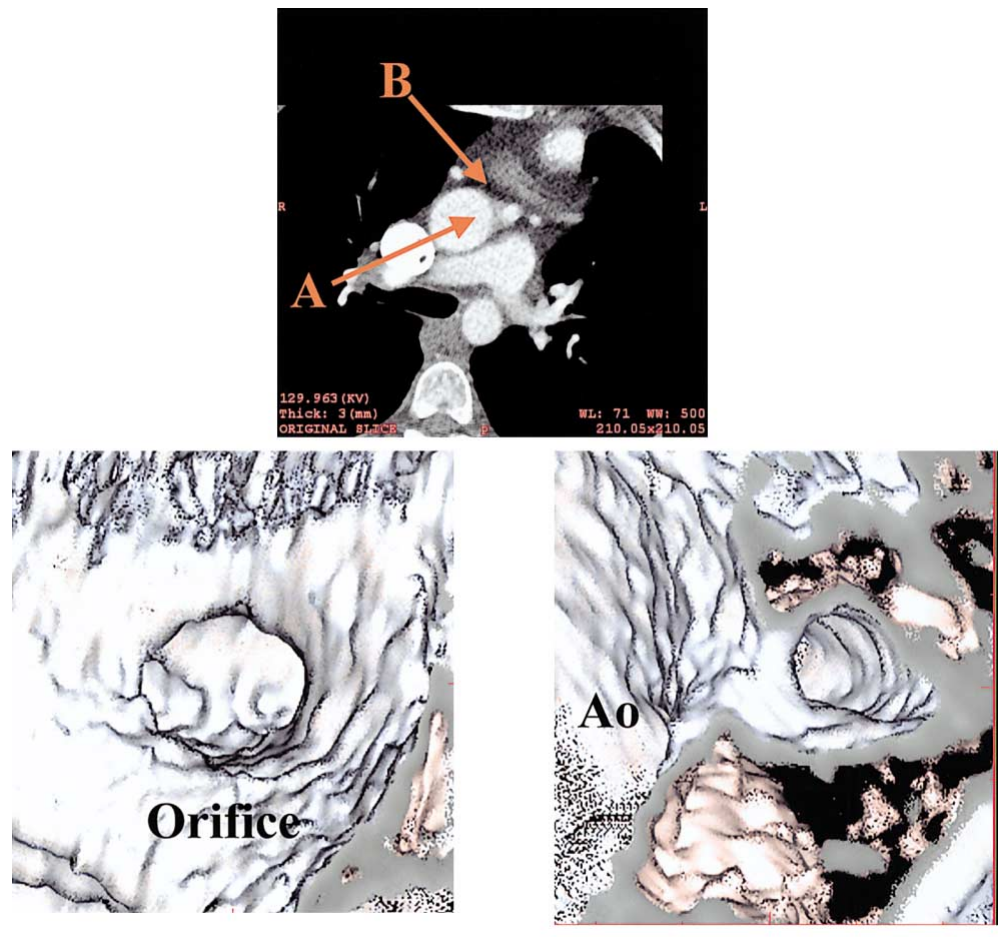

A

Fig 2. Axial source image at the level of the orifice of the left main trunk of the right coronary artery and left anterior descending branch. (A) Transverse images of the orifice of the left main trunk from direction ' $A$ ' showed that the shape of the orifice of the left main trunk was not slit-like and (B) Cut-plane volume rendering showed the longitudinal image of the left main trunk from direction 'B'. Ao, aorta. We eliminated visualization of the contrast medium from the lumen to show the inside of the lumen and the structures surrounding the lumen, which appeared in cut-away views to represent the vessel walls.

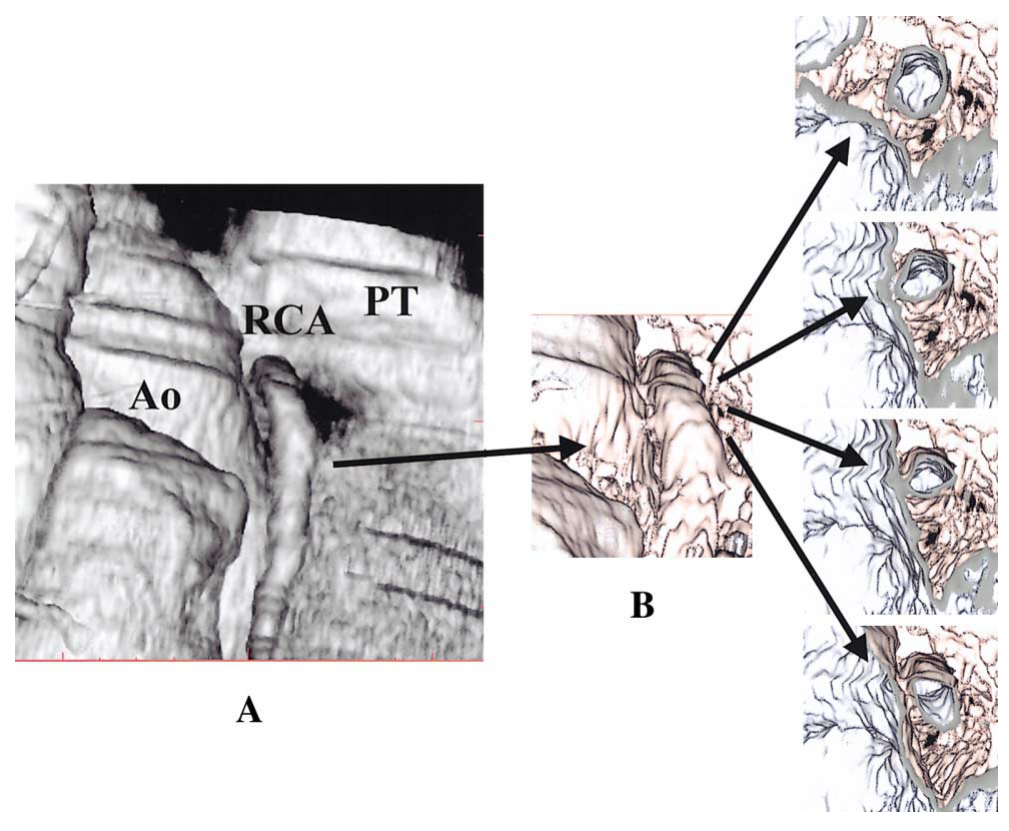

Fig 3. (A) The approximately 60-degree right anterior view volume rendering shows the lumen of the right coronary artery (RCA), ascending aorta (Ao) and pulmonary trunk (PT) filled with contrast medium. (B) Same view as (A) showing that the surface of the RCA vessel was thicker than the lumen. The shape of the opacity and color curves was the same as in Fig 2A,B. Cut-plane volume rendering showed the transverse image of the RCA and the longitudinal image of the ascending Ao and we could observe the inside of the lumen and the structure surrounding the lumen.

\section{Methods}

The subject was a 17-year-old male who had previously undergone the Rastelli operation for the third type of transposition of the great vessels. He had experienced no ischemic episode previously. EBCT (Imatron C-150XP, Imatron, South San Francisco, CA, USA) was performed in the single-slice step volume scan (SVS) mode during breathholding over $80 \mathrm{~mm}$ of the proximal coronary arteries using $3 \mathrm{~mm}$ collimation, $2 \mathrm{~mm}$ table incrementation, and triggered to $80 \%$ of the electrocardiogram (ECG) R-to-R interval (end-diastolic phase). EBCT was performed in conjunction with a 100-ml intravenous injection of iodinated contrast medium $(300 \mathrm{mgI} / \mathrm{ml})$ at $2.5 \mathrm{ml} / \mathrm{s}$ with a delay time of $25 \mathrm{~s}$ before the acquisition of data.

The reconstruction was performed with a viewing field of $21 \times 21 \mathrm{~cm}$; as a result, the voxel size of the in-plane became $0.41 \times 0.41 \mathrm{~mm}$ by a $512 \times 512$ matrix. These data were sent to a workstation (Advantage-Windows, General Electronics, Milwaukee, WI, USA) and reconstructed into 3-dimensional images through VR. 


\section{Results}

We obtained images without motion artifacts so the course of the RCA was able to evaluated accurately. In the axial source images, the RCA originated from the left sinus of Valsalva with the left main trunk and coursed between the ascending aorta and the PT (Fig 1). The angle of the bend at the bifurcation of the left main trunk was sharp.

Perspective VR showed the shape of the orifice of the left main trunk, which was not slit-like, and the cut-plane VR showed the spatial relationships not only between the lumen but also the surface of the vessels among the RCA, ascending aorta and the PT (Figs 2,3).

\section{Discussion}

Whether patients diagnosed with this anomaly will have acute ischemic episodes is difficult to predict. Clinical history, detailed angiographic study of the origin of the aberrant vessel accompanied by assessment of myocardial lactate metabolism during pacing, evaluation of coronary sinus blood flow during exercise, and response to maximal exercise testing were reported by Chaitman et al as all being of use in detecting patients predisposed to acute ischemic episodes!

In the present study we used EBCT because it achieves ultrafast scan speeds by sweeping a steered electron beam on a fixed tungsten target ring, thereby providing a moving $\mathrm{X}$-ray source without mechanical motion? EBCT acquisition is accomplished in $50 \mathrm{~ms}$ in the cine and flow modes or 100 $\mathrm{ms}$ in the SVS mode with high in-plane spatial resolution and a minimum slice thickness of $1.5 \mathrm{~mm}$ ? The SVS mode is used to define static anatomical information with matrix sizes of $512 \times 512$ with ECG gating during diastole, which minimizes blurring caused by cardiac motion. With those volumetric data, 3-dimensional images can be reconstructed by VR, which enables differences in objects to be distinguished through selection of the shape of opacity and color curves for CT numbers, and thus we could represent the structures surrounding the lumen (vessel walls and fatty tissue) as well as the vessel lumen filled with contrast medium ${ }^{10}$ Thus, the complex morphology of the anomalous RCA and its relationship to adjacent structures can be elucidated!1 We used the axial source images and VR images from EBCT to evaluate the course of the RCA, the angle of the bend at the bifurcation of the left main trunk, the shape of the orifice of the left main trunk and the spatial relationships of the main vessels.

The definition of an anomalous origin of a coronary artery is made on the basis that there are no other abnormalities present, so strictly speaking the present patient did not have an anomalous origin of a coronary artery because he had undergone the Rastelli procedure for the third type of transposition of the great arteries. However, the axial source images showed that the RCA originated from the left sinus of Valsalva with the left main trunk and coursed between the ascending aorta and the PT, so our study has still demonstrated the usefulness of 3-dimensional VR images in determining the possible cause of ischemia in patients with a truly anomalous coronary artery.

Perspective VR showed the shape of the orifice of the left main trunk, which was not slit-like, and the cut-plane VR showed the spatial relationship of the vessels. Because the patient had not had an ischemic episode and there was nothing except surrounding fat to compress the RCA on the anterior thoracic side, the hypothesis of exercise-induced ischemia caused by compression from the dilated aorta or the PT seems unlikely. With that hypothesis, either the RCA must come into contact with the ascending aorta or the PT and the area of the lumen of the RCA decrease or be disturbed so that it is extended by compression or the slitlike orifice of the left main trunk must be compressed so that closure or near closure is achieved. It is more likely that the angle of the bend at the bifurcation of the left main trunk became sharper during systole, thus causing ischemia during exercise when there is increased demand for oxygen.

The evidence provided by loaded radioisotope of an ischemic mechanism is only indirect. It will not show the RCA compressed by the dilated aorta or the PT because of exercise or a drug, but only the existence of ischemia. However, if the 3-dimensional images of EBCT data are combined with VR, when the slit-like orifice of the left main trunk is compressed to closure or near closure or the RCA is compressed by the aorta or the PT, there is the possibility of direct evidence of the ischemia being caused by the anomalous origin of coronary arteries.

These EBCT data sets were taken under resting conditions, but images can be obtained just after or during exercise, or loading with drug.2 We triggered the ECG at $80 \%$ of the R-to-R interval with a $100 \mathrm{~ms}$ scan time. As a result, the scan was performed at the end of diastole. Because the diameter of the thoracic aorta is maximal at end-systole and the amount of blood flow of the RCA is nearly equal during both systole and diastole, we consider that it is preferable to trigger at systole.

Recently, multislice computed tomography has become available with which retrospective ECG gated reconstruction can be achieved and which provides volume data for different cardiac phases. ${ }^{13}$ Observation of the RCA not only in the diastolic or systolic phase but also the throughout the cardiac cycle by a retrospective gating technique would be more informative.

\section{References}

1. Chaitman BR, Lesperance JL, Saltiel J, Bourassa MG: Clinical, angiographic and hemodynamic findings in patients with anomalous origin of the coronary arteries. Circulation 1976; 53: $122-131$

2. Kimbiris D, Iskandrian AS, Segal BL, Bemis CE: Anomalous aortic origin of coronary arteries. Circulation 1978; 58: 606-615

3. Roberts WC: Major anomalies of coronary arterial origin seen in adulthood. Am Heart J 1986; 111: $941-963$

4. Kragel AH, Roberts WC: Anomalous origin of either the right or left main coronary artery from the aorta with subsequent coursing between aorta and pulmonary trunk: Analysis of 32 necropsy cases. Am J Cardiol 1988; 62: 771 - 777

5. McConnell MV, Ganz P, Selwyn AP, Li W, Edelman RR, Manning WJ: Identification of anomalous coronary arteries and their anatomic course by magnetic resonance coronary angiography. Circulation 1995; 92: $3158-3162$

6. Post JC, van Rossum AC, Bronzwaer JGF, de Cock CC, Hofman $\mathrm{BM}$, Valk J, et al: Magnetic resonance angiography of anomalous coronary arteries: A new gold standard for delineating the proximal course? Circulation 1995; 92: 3163-3171

7. Koh KK: Confirmation of anomalous origin of the right coronary artery from the left sinus of Valsalva by means of transesophageal echocardiography. Am Heart J 1991; 122: 851-854

8. Gould RG: Principles of ultrafast computed tomography: Historical aspects, mechanism of action, and scanner characteristics. In: Stanford W, Rumberger J, editors. Ultrafast computed tomography in cardiac imaging: Principles and practice. New York: Futura, 1992: 1-15

9. Rumberger JA: Ultrafast computed tomography scanning modes, scanning planes and practical aspects of contrast administration. In: Stanford W, Rumberger J, editors. Ultrafast computed tomography in cardiac imaging: Principles and practice. New York: Futura, 1992: $17-24$ 
10. Funabashi N, Matsumoto A, Yoshida T, Watanabe S, Misumi K, Masuda Y: Usefulness of three-dimensional visualization of coronary arteries using electron-beam computed tomography data with volume rendering. Jpn Circ J 2000; 64: 644-646

11. Funabashi N, Rubin GD: Qualitative blood flow differentiation: Depiction of a left to right cardiac shunt across a ventricular septal defect using electron-beam computed tomography. Jpn Circ J 2000; 64: $901-903$
12. Chomka EV, Brundage BH: Left ventricular evaluation by exercise ultrafast computed tomography. In: Stanford W, Rumberger J, editors. Ultrafast computed tomography in cardiac imaging: Principles and practice. New York: Futura, 1992: 139-160

13. Klingenbeck-Regn K, Schaller S, Flohr T, Ohnesorge B, Kopp AF, Baum U: Subsecond multi-slice computed tomography: Basics and applications. Eur J Radiol 1999; 31: 110-124 